\title{
Virulência de isolados clínicos de Candida tropicalis
}

\author{
Virulence of clinical isolates of Candida tropicalis
}

Carlos Henrique Lopes Rocha1 ; Flaviane Maria Galvão Rocha1; Aline Michelle Silva Mendonça ${ }^{2}$; Eduardo Willian de Alencar Pereira ${ }^{3}$; Francisco Marcelo Costa da Silva ${ }^{4}$; Amanda Romana Santos da Silva ${ }^{1}$; Silvio Gomes Monteiro ${ }^{5}$; Cristina de Andrade Monteiro ${ }^{5}$

\begin{abstract}
Resumo: Verificar a produção enzimática e a capacidade de aderência e formação de biofilme em isolados clínicos de Candida tropicalis. 14 isolados provenientes de amostras clínicas obtidas de um laboratório particular de São Luís- MA, foi realizada a identificação pelo sistema automatizado VITEK (bio Mérieux). Todos os isolados foram aderentes ao vidro e a maioria apresentou aderência moderada, $42,9 \%$ foram positivas para proteinases, $78,6 \%$ para fosfolipases, com $\mathrm{Pz}$ variando de 0,47 a 0,9 . Todos os isolados apresentaram atividade hemolítica em meio sólido e sete isolados mostraram $\mathrm{IH} \geq 2.0$. Os isolados de Candida tropicalis avaliados foram capazes de aderir a superfícies inertes com padrões distintos de organização celular, onde alguns destes tipos poderiam facilitar a formação de biofilmes por estas espécies, a propriedade de aderência não está diretamente relacionada ao sítio de infecção, mas provavelmente com fatores específicos destes microrganismos e com a resposta do hospedeiro.
\end{abstract}

Palavras chaves: Candida tropicalis, adesão, virulência, exoenzimas

\begin{abstract}
To verify the enzymatic production and the adhesion and biofilm formation capacity in clinical isolates of Candida tropicalis. 14 isolates from clinical specimens obtained from a private laboratory in São Luís, Brazil, were identified by automated VITEK (bio Mérieux). All the isolates were adherent to the glass and the majority presented moderate adherence, $42.9 \%$ were positive for proteinases, $78.6 \%$ for phospholipases, with $\mathrm{Pz}$ ranging from 0.47 to 0.9 . All isolates showed hemolytic activity in solid medium and seven isolates showed $\mathrm{HI} \geq 2.0$. Candida tropicalis isolates were able to adhere to inert surfaces with distinct patterns of cellular organization, where some of these types could facilitate the formation of biofilms by these species, the adhesion property is not directly related to the site of infection, but probably with specific factors of these microorganisms and the response of the host.
\end{abstract}

Key words: Candida tropicalis, adhesion, virulence, exoenzymes

\footnotetext{
${ }^{1}$ Discente do Programa de Mestrado em biologia Parasitária- laboratório de Microbiologia aplacada da Universidade CEUMA - Campus Renascença. Universidade CEUMA, Maranhão

2 Discente do curso em Biomedicina da Universidade CEUMA - Campus Renascença. Universidade CEUMA, Maranhão

${ }^{3}$ Discente da especialização em Microbiologia Clínica da Universidade CEUMA - Campus Renascença. Universidade CEUMA, Maranhão

${ }^{4}$ Discente do curso de Licenciatura em biologia do IFMA - Campus Monte Castelo

${ }^{5}$ Docente Permanente do Programa de Mestrado em Biologia Parasitária da Universidade CEUMA - Campus Renascença. Universidade CEUMA, Maranhão
} 


\section{Introdução}

O aumento da incidência das infecções fúngicas tem sido associada a pacientes com comprometimento do sistema imunológico ${ }^{1,2,3}$, Candida albicans é a espécie frequentemente isolada ${ }^{4,5,2,3}$. No entanto, outras espécies têm aumentado significativamente sua prevalência, dentre estas $C$. tropicalis ${ }^{6,3,7}$. As infecções por $C$. tropicalis são comumente associadas a malignidade, com alguns estudos relatando sua alta incidência entre pacientes com doenças hematológicas, como por exemplo, leucemia mieloide aguda $8,9,10,11$, sendo causa comum de infecção em unidades de terapia intensiva, é um problema particular em pacientes neutropênicos ${ }^{12,13,14}$. A mortalidade associada à candidemia por $C$. tropicalis nessas populações varia de 30 a $70 \%$, com as maiores taxas observadas entre os idosos $8,9,15,11,16$.

Os principais fatores de virulência de Candida associados às infecções sanguíneas incluem produção de hemolisina, produção de proteinases e formação de biofilmes ${ }^{17,18,19}$. A formação de biofilmes, embora presente em todas as espécies, difere significativamente entre elas, dependendo da superfície, por exemplo, e das suas características individuais. O biofilme de $C$. tropicalis corresponde a uma rede de leveduras, pseudo hifas e hifas ${ }^{20} . A$ análise da propriedade de aderência das espécies de Candida e de seus fatores de virulência contribui para a compreensão do comportamento destas em um processo de infecção ${ }^{21,22}$.

As fosfolipases estão relacionadas com o processo de ruptura da membrana das células do hospedeiro no momento da invasão ${ }^{25}$. Constituem um grupo heterogêneo de enzimas com capacidade para hidrolisar um ou mais ésteres ligados a glicerofosfolipídeos ${ }^{23}$, quando promovem a clivagem dos fosfolipídeos, ocorre a instabilidade da membrana, com subsequente lise celular ${ }^{25}$. Cada enzima age clivando um éster específico, em geral, encontradas na periferia das células de leveduras, sendo secretadas no ambiente externo, na forma de hifas concentram-se nas extremidades ${ }^{24}$.

As proteinases clivam proteínas relacionadas com o mecanismo de defesa do hospedeiro. Podem facilitar a colonização, penetração e invasão de Candida spp., durante o processo de infecção SAPs promovem a digestão de proteínas, evasão das defesas por degradação de imunoglobulinas e proteínas do sistema complemento, aderência e degradação das barreiras do hospedeiro promovendo a inativação dos inibidores da protease sérica ${ }^{26,27}$.

A atividade hemolítica corresponde a um fator de virulência de alguns microrganismos que torna possível o crescimento dos mesmos no hospedeiro pela utilização de proteínas ligadas ao ferro, leveduras do gênero Candida tem a capacidade de secretar um fator hemolítico conhecido como hemolisina, promovendo a liberação da hemoglobina, utilizada como fonte de ferro ${ }^{28}$.

Estas exoenzimas bem como biofilmes são frequentemente estudados na espécie $C$. albicans que desempenham papel central na patogenicidade da espécie $^{29}$. Assim, a produção e secreção dessas exoenzimas e capacidade de adesão e biofilmes de $C$. tropicalis é pouco conhecida. Por esta razão, o objetivo do presente trabalho foi verificar a produção enzimática e a capacidade de aderência e formação de biofilme em isolados clínicos de Candida tropicalis provenientes de diferentes sítios anatômicos.

\section{Material e Métodos}

Foram utilizados neste estudo um total de 14 isolados de Candida tropicalis 
provenientes de várias amostras clínicas de diferentes sítios anatômicos como secreções vaginais, urina, sangue, secreções traqueais e de ponta de cateteres, todas obtidas de um laboratório particular da cidade de São Luís - MA, gentilmente cedidas e depositadas no Laboratório de Micologia Médica da Universidade Ceuma e 0 isolado de referência C. albicans ATCC18804 (American Type Culture Collection, Rockville, Md) foi utilizado em todos os experimentos como controle. Todos os isolados clínicos foram previamente identificados pelo sistema automatizado VITEK (BioMérieux), foram mantidas a $20^{\circ} \mathrm{C}$ em caldo BHI (Brain Heart InfusionAcumedia Manufactures).

\section{Aderência em material inerte}

Para determinar a capacidade de adesão foi utilizado 0 protocolo de Menezes et $\mathrm{al}^{30}$. Resumidamente, foram utilizadas microplacas de poliestireno de 24 poços (Zellkultur Test plate 24, Techno Plastic Products), contendo em cada poço uma lamínula redonda estéril de vidro (Glasscyto). Foram adicionados $40 \mu \mathrm{l}$ do inóculo padronizado a $10^{6} \mathrm{UFC} / \mathrm{mL}$ mais $960 \mu \mathrm{L}$ de meio $\mathrm{BHI}$ e incubados a $37^{\circ} \mathrm{C}$ por 18 horas. Após incubação o meio foi removido e a microplaca foi lavada com água destilada estéril para remoção das células não aderentes. Foi adicionado cristal violeta a $1 \%$ às lamínulas por 5 minutos, em seguida foram lavadas duas vezes com água estéril para remoção do excesso de corante e colocadas em lâminas para observação em microscopia de luz (NIKON Eclipse E100). A determinação da aderência foi realizada contando-se o número de leveduras aderidas. A classificação da capacidade de adesão das células em lamínulas foi realizada considerando a contagem de células aderidas por campo. Para cada lamínula do ensaio foi analisado um máximo de 70 campos. O número de células obtido foi dado como média de três experimentos realizados para cada isolado. A capacidade de adesão foi classificada como negativa quando menos que 1 (uma) célula estava aderida por campo, visualizados 70 (setenta) campos da lamínula; fraca quando entre 1 a 10 (uma a dez) células aderidas por campo em 50 (cinquenta) campos observados; moderada quando mais de 10 (dez) células por campo aderidas em 30 (trinta) campos e forte quando igual ou maior que 25 (vinte e cinco) células aderidas por campo em 20 (vinte) campos analisados.

\section{Formação de biofilme}

Para a formação de biofilme seguiuse 0 protocolo de Menezes et $\mathrm{al}^{30}$. Resumidamente, $20 \mu \mathrm{l}$ de suspensão celular na concentração de $10^{6} \mathrm{UFC} / \mathrm{mL}$ foram adicionados a microplacas de 96 poços acrescidos de $180 \mu \mathrm{L}$ de $\mathrm{BHI}$ suplementado com $6 \%$ de glicose, incubadas a $37^{\circ} \mathrm{C}$ por 24 horas. Após a incubação foi removido o conteúdo de cada poço, a microplaca foi lavada três vezes com água destilada estéril e foi acrescentado violeta cristal (1\%). As microplacas foram novamente lavadas para remoção do excesso do corante e por fim foram adicionados $200 \mu \mathrm{L}$ de água destilada a cada poço para leitura de absorbância a $450 \mathrm{~nm}$ em leitor de microplaca. Os valores de absorbância foram dados como média de três (03) experimentos para cada isolado e os dados foram expressos em porcentagem. A produção de biofilme foi classificada em negativa $(\% A<5)$, fraco $(+1 ; 5 \leq \% A<$ 20), moderada (+2; $20 \leq \% A<35)$, forte $(+3 ; 35 \leq \% \mathrm{~A}<50)$ e muito forte $(+4 ; \% \mathrm{~A}$ $\geq 50$ ). 


\section{Produção de exoenzimas}

O teste de hemolisina foi realizado de acordo com um protocolo de Luo et al. ${ }^{31}$, considerando algumas adaptações de Branco et al. ${ }^{32} \mathrm{Em}$ resumo, inicialmente 7 $\mathrm{mL}$ de sangue de carneiro desfibrinado foi centrifugado a 3000 rpms por 5 minutos e - sobrenadante foi descartado. Aos eritrócitos foram acrescentados $10 \mathrm{~mL}$ de tampão fosfato-salina (PBS) e lavados três vezes por centrifugação. Os eritrócitos foram ressuspendidos em $5 \mathrm{~mL}$ de PBS em seguida adicionados a $100 \mathrm{~mL}$ de SDA acrescido de cloranfenicol e $3 \%$ de glicose. Uma suspensão celular $1 \times 10^{6}$ células / ml e $10 \mu \mathrm{l}$ foi inoculado, as placas foram incubadas a $37^{\circ} \mathrm{C}$. Os diâmetros da colônia e $O$ halo transparente foram medidos. A intensidade da hemólise foi obtida pela razão: colônia / halo + colônia. A classificação foi feita de acordo com o Índice Hemolítico ( $\mathrm{IH})$ : positiva $(1,00<\mathrm{lH}<1,5) ; \quad$ fortemente positiva $(\mathrm{IH}>1,5)$.

A determinação da produção de proteinases foi realizada de acordo com Aoki et al. ${ }^{33} \mathrm{O}$ meio teste consistiu de placas com Agar contendo albumina bovina de soro (BSA). Sessenta mililitros de uma solução contendo 0.04 gMgSO4.7H2O, $0.5 \mathrm{~g} \mathrm{K2HPO} 4,1 \mathrm{~g} \mathrm{NaCl}$, $0.2 \mathrm{~g}$ de extrato de levedura, $4 \mathrm{~g}$ glicose $\mathrm{e}$ $0.5 \mathrm{~g}$ BSA (Bovine Serum Albumin Fraction V, Sigma Chem Co., St. Louis, Mo., USA) foram preparados, e o $\mathrm{pH}$ ajustado para 4.0. A solução foi esterilizada por filtração e então acrescentada a $140 \mathrm{ml}$ de Agar fundido. A suspensão celular padronizada foi inoculada e as placas foram incubadas a $37{ }^{\circ} \mathrm{C}$ por 7 dias. O valor da zona de precipitação $(\mathrm{Pz})$ foi dado como a média dos diâmetros avaliados (colônia / halo + colônia), PZ foi classificada de acordo com Price et al. ${ }^{34}$, sendo Pz: muito forte ++++ $(P z \leq 0,69)$, forte $+++(P z$ entre 0,70-0,79), média $++(P z$ entre $0,80-0,89)$ ou fraca + (Pz entre 0,90-0,99).

A produção de fosfolipases dos isolados foi realizada de acordo com o método em placa com gema de ovo de Price et al. $^{34}$. O meio teste consistiu de SDA contendo $1 \mathrm{M}$ de cloreto de sódio, $0.005 \mathrm{M}$ de cloreto de cálcio e $2 \%$ de gema de ovo. Três microlitros da suspensão foram inoculados no meio. As placas foram incubadas a $37^{\circ} \mathrm{C}$ e os diâmetros das colônias e das zonas de precipitação mais a colônia foram medidos após 7 dias de inoculação. A presença da enzima fosfolipase foi observada pela formação de um halo ao redor da colônia. Medidas e cálculos da zona de atividade enzimática foram realizadas como descrito anteriormente ${ }^{34}$. Os coeficientes de $\mathrm{Pz}$ foram utilizados para classificar a produção enzimática sendo $\mathrm{Pz}=1$ negativo; entre $0.90-0,99(+)$, baixo; entre 0.80-0.89 (+ $+)$; 0.70-0.79 (+ + +) alto; e $<0.70(++++)$ muito alto.

\section{Análise estatística}

Os dados foram analisados através do programa estatístico Bioestat 5.0 (2007). A normalidade da variável numérica absorbância foi analisada através do teste de Lilliefors. Como a distribuição não foi normal, ela foi analisada através de teste não paramétrico. Juntamente com as variáveis ordinais, a classificação do nível de biofilme e da adesão em relação à espécie e ao sítio foram feitas através do teste não paramétrico de Kruskal Wallis e a análise post-hoc foi feita pelo teste StudentNewman-Keulls. O nível de significância (a) aplicado em todos os testes foi de $5 \%$, ou seja, considerou-se significativo quando $p<0,05$.

\section{Resultados}


Os testes mostraram que todos os isolados de $C$. tropicalis foram aderentes ao vidro e a maioria apresentou aderência moderada (7 isolados) (Figura 1). Apenas um isolado proveniente de urina e outro de sangue mostraram aderência forte ao vidro. A maioria $(n=10)$ dos isolados foi produtor de biofilme moderado. Dois isolados, um proveniente de urina e outro de secreção traqueal, não foram produtores de biofilme. Um isolado proveniente de sangue foi classificado como formador forte de biofilme. Não foi observada diferença estatística significativa em relação à origem do sítio anatômico e a capacidade dos isolados de formarem biofilme $(p<0.05)$.

Figura 1. Aderência de isolados de $C$. tropicalis a vidro. Em A, exemplo de aderência considerada moderada; em B, exemplo de aderência forte.

A

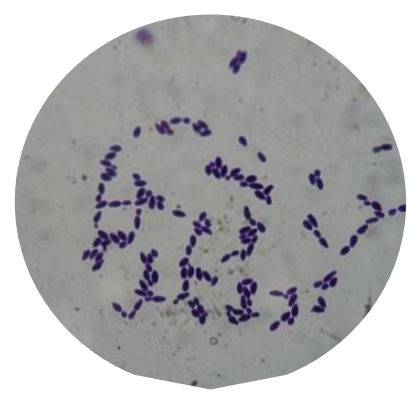

B

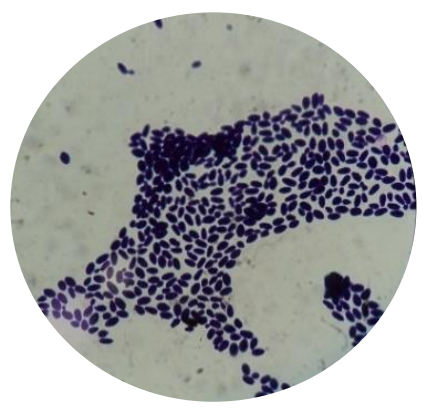

Para a produção de proteinases $42,9 \%$ das amostras foram positivas (Tabela 1), todas foram classificadas como boas produtoras com $\mathrm{Pz}$ variando de 0,56 (para um isolado de urina) a 0,86 (isolado de sangue) (Tabela 1; Figura 3). Após os dados serem analisados estatisticamente, não houve diferença entre a produção de proteinases entre os isolados provenientes dos diferentes sítios anatômicos $(p>0,05)$.

Tabela1. Atividade de proteinase de isolados clínicos de Candida tropicalis.

\begin{tabular}{cccc}
\hline C. tropicalis & $\begin{array}{c}\text { Sítio de } \\
\text { origem }\end{array}$ & PZ & Resultado \\
\hline 8 & Urina & 0,73 & 3 \\
82 & Urina & 1 & - \\
84 & Sec. Traqueal & 0,61 & 4 \\
63 & Sangue & 1 & - \\
92 & Sec. Traqueal & 1 & - \\
51 & Sec. Traqueal & 0,69 & 4 \\
39 & Urina & 0,56 & 4 \\
64 & Urina & 0,63 & 4 \\
95 & Sangue & 0,86 & 2 \\
59 & Urina & 1 & - \\
103 & Sec. Traqueal & 1 & - \\
85 & Sec. Traqueal & 1 & - \\
C. albicans & Isolado de & 0,7 & 3 \\
\hline \multicolumn{4}{r}{ referência } \\
\hline
\end{tabular}

Em relação à produção de fosfolipases, 78,6\% amostras foram positivas com $\mathrm{Pz}$ variando de 0,47 a 0,9 (Tabela 2; Figura 3).

Todos os isolados de C. tropicalis apresentaram atividade hemolítica em meio sólido e sete isolados mostraram $\mathrm{IH} \geq$ 2.0 (Figura 4). Não foi encontrada diferença significativa em nenhum dos sítios em relação à produção de hemolisinas $(p>0,05)$. 


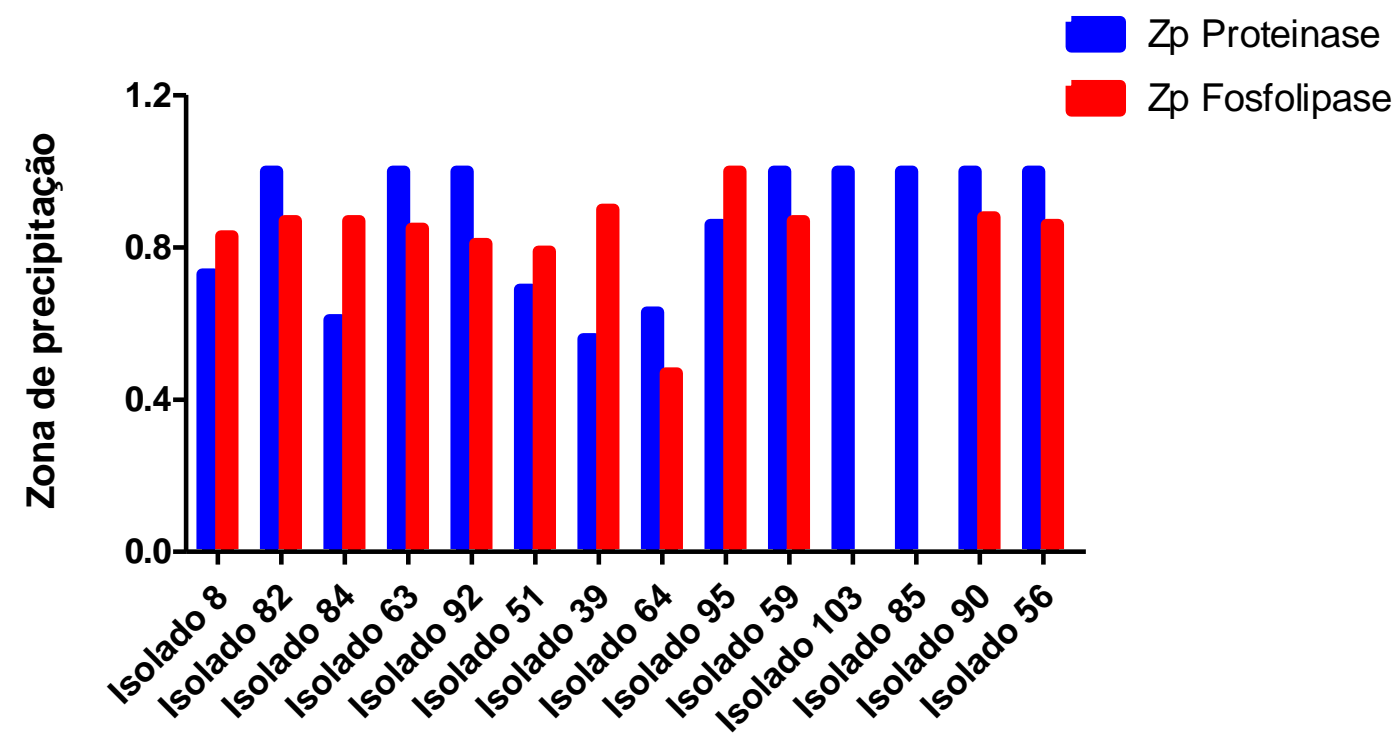

Isolados de $C$. tropicalis

Figura 3. Atividade de proteinases e de fosfolipases de isolados de Candida tropicalis obtidos de diversos sítios anatômicos.

- Índice hemolítico

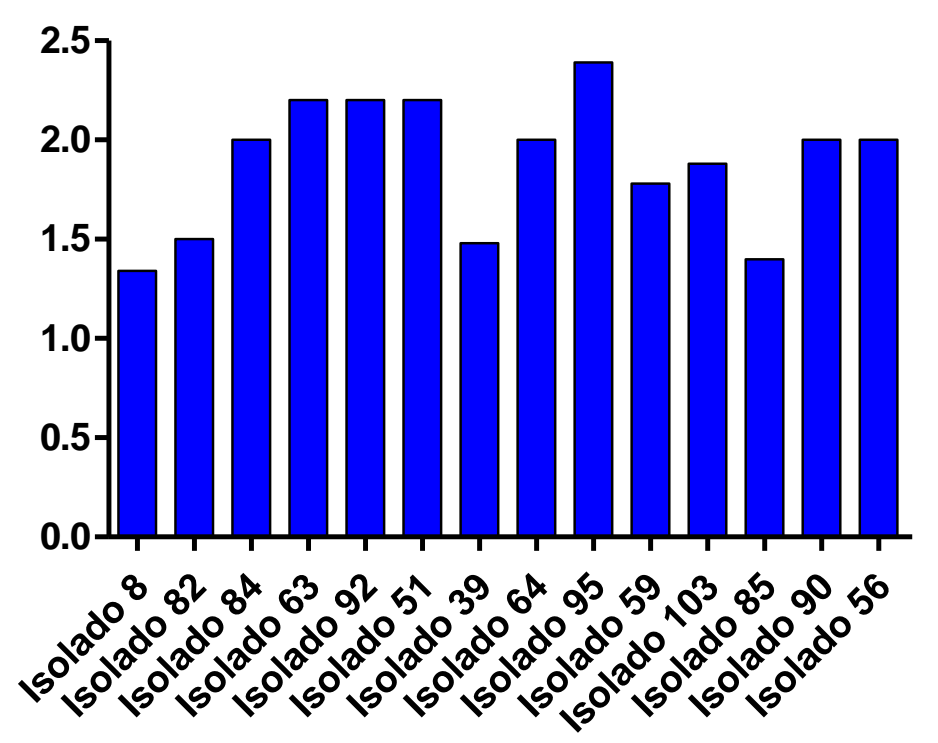

Isolados de $C$. tropicalis 
Figura 4. Atividade hemolítica de isolados de Candida tropicalis obtidos de diversos sítios anatômicos.

Tabela 2 Atividade de fosfolipase de isolados clínicos de Candida tropicalis obtidos de diversos sítios anatômicos.

\begin{tabular}{cccc}
\hline $\begin{array}{c}C . \\
\text { tropicalis }\end{array}$ & $\begin{array}{c}\text { Sítio de } \\
\text { origem }\end{array}$ & $\mathrm{PZ}$ & Resultado \\
\hline 90 & Urina & 0,88 & 2 \\
59 & Urina & 0,87 & 2 \\
82 & Urina & 0,87 & 2 \\
84 & Sec. Traqueal & 0,87 & 2 \\
63 & Sangue & 0,85 & 2 \\
56 & Sec. Traqueal & 0,86 & 2 \\
8 & Urina & 0,83 & 2 \\
92 & Sec. Traqueal & 0,81 & 2 \\
51 & Sec. Traqueal & 0,79 & 3 \\
39 & Urina & 0,9 & 2 \\
64 & Urina & 0,47 & 4 \\
95 & Sangue & 1 & - \\
C. & Isolado de & 0,7 & 3 \\
albicans & referência & 0,7 \\
\hline \multicolumn{4}{c}{} \\
\hline
\end{tabular}

\section{Discussão}

Aderência de Candida às superfícies celulares do hospedeiro é essencial para que ocorra a colonização e posterior infecção ${ }^{35}$. Alguns estudos relatam que esta capacidade pode variar entre as espécies $^{36,37}$. Este fato explica porque algumas espécies colonizam mais frequentemente as superfícies mucosas do que outras e a capacidade de aderir a materiais inertes pode ser um indicativo de provável aderência a células vivas ${ }^{36}$. Nesta pesquisa, todos os isolados de C. tropicalis aderiram a lamínulas de vidro, corroborando com outros estudos que relatam a aderência de espécies de Candida a materiais inertes como silicone ${ }^{36}$ e vidro ${ }^{38}$. A elevada capacidade de aderência por C. tropicalis pode indicar uma virulência aumentada desta espécie em relação às demais, corroborando com resultados obtidos por alguns trabalhos que mostraram que espécies de CNA tem aumentado esta capacide ${ }^{39,32}$.

O aumento da frequência de espécies de CNA isoladas de diversos sítios do corpo e de dispositivos hospitalares é um fato que deve ser ressaltado, tendo em vista que muitos estudos tendem a centrar a preocupação exclusivamente em $C$. albicans e menos importância tem sido dada a outras espécies. A detecção de $C$. tropicalis está normalmente associada à infecção, diferente de $C$. albicans que está associada à microbiota normal. Todavia, em diversos trabalhos $C$. tropicalis foi a segunda espécie mais isolada de pacientes saudáveis, perdendo somente para C. albicans ${ }^{32,40}$. A produção de proteinase é considerada um importante fator de virulência porque aumenta a capacidade do microrganismo colonizar e penetrar o tecido do hospedeiro, além de destruir um número significativo de importantes proteínas como imunoglobulinas, proteínas do sistema complemento e citocinas. Neste estudo os isolados positivos de C. tropicalis analisados apresentaram boa produção de proteinase, corroborando com Oliveira et al. ${ }^{40}$, em seu estudo $C$. tropicalis destaca-se dentre as CNA.

No presente estudo os isolados foram bons produtores de 
fosfolipases. A fosfolipase está relacionada à maior capacidade de invadir o tecido, dentre várias funções destacam-se: penetração na célula do hospedeiro e adesão às células epiteliais, C. tropicalis é conhecida por ter reduzida capacidade de produção desta enzima, embora alguns autores relatem que a produção de fosfolipase extracelular é fortemente dependente da linhagem, - que diverge dos resultados aqui encontrados. Porém o trabalho de D'eça Júnior et al. ${ }^{39}$ corroboram os dados desta pesquisa mostrando que isolados de $C$. tropicalis são bons produtores de fosfolipases.

Quanto à hemolisina, sabe-se que esta facilita a invasão de hifas na mucosa de pacientes com candidíase disseminada e tem capacidade de lisar eritrócitos em busca de ferro para favorecer seu crescimento e disseminação, podendo causar anemia e déficit de transporte de oxigênio nos pacientes. Assim, a produção desta enzima está relacionada à maior virulência dos isolados 19. Pode ser observado neste trabalho nos resultados obtidos com os isolados de $C$. tropicalis aqui avaliados. Todas as amostras demonstraram capacidade hemolítica com índices hemolíticos relativamente altos quando comparados com o que se tem na literatura ${ }^{39,32}$. Em um estudo realizado por França et al. ${ }^{40} \mathrm{com} 28$ amostras clínicas de C. tropicalis, todas apresentaram atividade hemolítica, resultado semelhante ao encontrado neste trabalho.

Em síntese, os resultados obtidos nesta pesquisa demonstram que os isolados de Candida tropicalis avaliados foram capazes de aderir a superfícies inertes se organizando em padrões distintos de organização celular, onde alguns destes tipos poderiam facilitar a formação de biofilmes por estas espécies. Os dados aqui obtidos também mostram que a propriedade de aderência não está diretamente relacionada ao sítio de infecção, mas provavelmente com fatores específicos destes microrganismos e com a resposta do hospedeiro.

\section{Referências}

1. Ortega M., Marco F., Soriano A., Almela M., Martínez J. A., Pitart C., et al. Candida spp. bloodstream infection: influence of antifungal treatment on outcome. J. Antimicrob. Chemother. 2010; 65: 562-568.

2. Junqueira J. C., Vilela S. F., Rossoni R. D., Barbosa J. O., Costa A. C. B. P., Rasteiro V. M. C., et al. Oral colonization by yeasts in HIV-positive patients in Brazil. Rev. Inst. Med. Trop. Sao Paulo. 2012. 54; 17-24.

3. Li Y. Y ., Chen W. Y., Li X., Li H. B., Li H. Q., Wang L., et al. Asymptomatic oral yeast carriage and antifungal susceptibility profile of HIV-infected patients in Kunming, Yunnan Province of China. BMC Infect. Dis. 2013. 13;46-53.

4. Delgado A. C., de Jesus Pedro R., Aoki F. H., Resende M. R., Trabasso P., Colombo A. L., et al. Clinical and microbiological assessment of patients with a long-term diagnosis of human immunodeficiency virus infection and Candida oral colonization. Clin. Microbiol. Infect. 2009. 15; 364-371.

5. Hise A. G., Tomalka J., Ganesan S., Patel K., Hall B. A., Brown G. D., et al. An essential role for the NLRP3 inflammasome in host defense against the human fungal pathogen Candida 
albicans. Cell Host Microbe. 2009. 5; 487-497.

6. Sant'Ana P. L., Milan E. P., Martinez R., Telles F. Q., Ferreira M. S., Alcântara A. P., et al. Multicenter Brazilian study of oral Candida species isolated from AIDS patients. Mem. Inst. Oswaldo Cruz. 2002. 97; 253-257.

7. Kaur R., Dhakad M. S., Goyal R., Haque A., Mukhopadhyay G. Identification and Antifungal susceptibility testing of Candida species: a comparison of Vitek-2 system with conventional and molecular methods. J. Glob. Infect. Dis.2016. 8;139-146.

8. Weinberger M., Leibovici L., Perez S., Samra Z., Ostfeld I., Levi I., et al. Characteristics of candidaemia with Candida albicans compared with nonalbicans Candida species and predictors of mortality. J. Hosp. Infect. 2005. 61; 146-154.

9. Nucci M, Colombo AL. Candidemia due to Candida tropicalis: clinical, epidemiologic, and microbiologic characteristics of 188 episodes occurring in tertiary care hospitals. Diagn Microbiol Infect Dis. 2007. 58; 77-78.

10. Tang J. L., Kung H. C., Lei W. C., Yao M., Wu U. I., Hsu S. C., et al. High incidences of invasive fungal infections in acute myeloid leukemia patients receiving induction chemotherapy without systemic antifungal prophylaxis: a prospective observational study in Taiwan. PLoS ONE (2015).10; 128- 410.

11. Cornely O. A., Gachot B., Akan H., Bassetti M., Uzun O., Kibbler C., et al. . Epidemiology and outcome of fungemia in a cancer Cohort of the Infectious Diseases Group (IDG) of the European Organization for Research and Treatment of Cancer
(EORTC 65031). Clin. Infect. Dis.2015.61; 324-331.

12. Negri $M$, Silva $S$, Henriques $M$, Oliveira R. Insights into Candida tropicalis nosocomial infections and virulence factors. Eur J Clin Microbiol Infect Dis. 2012. 31;1399-1412.

13. Silva $S$, Negri $M$, Henriques $M$, Oliveira R, Williams DW, Azeredo J. Candida glabrata, Candida parapsilosis and Candida tropicalis: Biology, epidemiology, pathogenicity and antifungal resistance. FEMS Microbiol Rev. 2012. 36; 288-305.

14. Turner, S. A., \& Butler, G. The Candida Pathogenic Species Complex. Cold Spring Harbor Perspectives in Medicine.2014. 4(9).

15. Morii D., Seki M., Binongo J. N., Ban R., Kobayashi A., Sata M., et al. Distribution of Candida species isolated from blood cultures in hospitals in Osaka, Japan. J. Infect. Chemother. 2014. 20; 558-562.

16. Wang E., Farmakiotis D., Yang D., McCue D. A., Kantarjian H. M., Kontoyiannis D. P., et al. The everevolving landscape of candidaemia in patients with acute leukaemia: nonsusceptibility to caspofungin and multidrug resistance are associated with increased mortality. J. Antimicrob. Chemother. 2015; 70.

17. Calderone RA, Fonzi WA. Virulence factors of Candida albicans. Trends Microbiol. 2001. 9; 327-335.

18. Lim C. S., Rosli R., Seow H. F., Chong P. P. Candida and invasive candidiasis: back to basics. Eur. J. Clin. Microbiol. Infect. Dis. 2012. 31;21-31.

19. Seneviratne, C. J., Rajan, S., Wong, S. S. W., Tsang, D. N. C., Lai, C. K. C., Samaranayake, L. P., \& Jin, L. Antifungal Susceptibility in Serum and Virulence Determinants of Candida Bloodstream Isolates from 
Hong Kong. Frontiers in Microbiology. 216. 7.

20. Bizerra FC, Nakamura CV, de Poersch C, Estivalet Svidzinski TI, Borsato Quesada RM, Goldenberg S, et al. Characteristics of biofilm formation by Candida tropicalis and antifungal resistance. FEMS Yeast Res. 2008.

21.Ramesh N, Priyadharsini $M$, Sumathi CS, Balasubramanian V, Hemapriya J, Kannan R. Virulence Factors and Anti Fungal Sensitivity Pattern of Candida sp. isolated from HIV and TB Patients. Indian J Microbiol. 2011.51; 273-278.

22. Douglas LJ. Biofilmes de Candida e seu papel na infecção. Trends Microbiol. 2003. 11.

23. Ombrella AM, Racca L, Ramos L. Actividades proteinasa y fosfolipasa de aislamientos de Candida albicans provenientes de secreciones vaginales con distintos valores de $\mathrm{pH}$. Rev Iberoam Micol. 2008. 25;12-16.

24. D'eça Júnior $A$. Atividade in vitro de fosfolipases, proteinases ácidas e hemolisinas de isolados clínicos de Candida. São Luís [dissertação de Mestrado em Biologia Parasitária Universidade Ceuma, 2010.

25. Mohan V, Ballal M. Proteinase and phospholipase activity as virulence factors in Candida species isolated from blood. Rev Iberoam Micol. 2008. 25; 208-210.

26. Taylor PR, Tsoni SV, Willment JA, Dennehy $\mathrm{KM}$, Rosas $\mathrm{M}$, Findon $\mathrm{H}$, Haynes K, Steele C, Botto M, Gordon $\mathrm{S}$, Brown GD. Dectin-1 is required for beta-glucan recognition and control of fungal infection. Nat Immunol. 2007. 8; 31-8.

27. Costa CR. Fatores de virulência de isolados de Candida de pacientes imunocomprometidos. Caracterização molecular de $C$. albicans susceptíveis e resistentes ao fluconazol. Goiás
[Tese de doutorado em Medicina Tropical - UFG Instituto de Patologia Tropical e Saúde Pública], 2009.

28. Rörig KCO, Colacite J, Abegg MA. Produção de fatores de virulência in vitro por espécies patogênicas do gênero Candida. Rev Soc Bras Med Trop. 2009. 42: 225-227.

29. Sandovsky-Losica H, Segal E. Infection of HEp-2 epithelial cells with Candida albicans: adherence and postadherence events. FEMS Immunol Med Microbiol. 2006. 46; 470-475.

30. Menezes, V.M., Vale, I.N.F., Monteiro, S.G., Gonçalves, L.H.B., Figueiredo, P.M.S., Andrade-Monteiro, C. Classificação da capacidade de Adesão de isolados clínicos de Candida Spp Em Padrões de arranjos celulares distintos. Rev. Patol. Tropical. 2013.42;3.

31. Luo G, Samaranayake LP, Yau JYY. Candida Species Exhibit differential In Vitro Hemolytic Activities. J Clin Microbiol. 2001.39; 2971-2974.

32. Castelo-Branco, P.V.G., Anjos, D.C.V. Nascimento, F.B., Vale, I.N.F, Azevedo, C.M.P.e S., Monteiro, S.G., Figueiredo, P.M.S., Andrade-Monteiro, C. Prevalência e Produção de exoenzimas por espécies de Candida Provenientes Da Mucosa Bucal De Pacientes Com AIDS E Indivíduos Hígidos. Rev. Patol. Tropical. 2012. 41 (4).

33.Aoki S, Ito-Kuwa S, Nakamura Y, Masuhara T. Comparative pathogenicity of wild-type strains and respiratory mutants of Candida albicans in mice. Zentralbl Bakteriol. 1990. 273; 332-343.

34. Price et al. Price MF, Wilkinson Ld, Gentry LO. Plate method for detection of phospholipase activity in Candida albicans. Sabouraudia.1982. 20; 7-14. 
35. Silva $S$, Negri $M$, Henriques $M$, Oliveira R, Williams D, Azeredo J. Adherence and biofilm formation of non-Candida albicans species. Trends Microbiol. 2011.19; 241-247.

36. Silva SCM. Virulence factors of nonCandida albicans species. [dissertation for Phd degree in Biomedical Engineering Universidade do Minho]. Escola de Engenharia, 2010.

37.Tamura NK, Gasparetto A, Svidzinski TI. Evaluation of the adherence of Candida species to urinary catheters. Mycopathologia. 2003.156; 269-272.

38. Menezes, V.M., Vale,I.N.F., Monteiro, S.G., Gonçalves, L.H.B., Figueiredo, P.M.S., Andrade-Monteiro, C. Classificação da capacidade de adesão de isolados clínicos de Candida spp em padrões de arranjos celulares distintos. Rev. Patol. Tropical. 2013. 42; 3.

39. D'EÇA JÚNIOR, A., Silva, A.F., ROSA, F.C., Monteiro, S.G., FIGUEIREDO, P.M.S., Monteiro, C. A. In vitro differential activity 0 fphospholipases and acid proteinases of clinical isolates of Candida. Rev Soc Bras Med Trop. 2011. 44; 334338.

40.França EJG, Favero D, Scremin $H$, Oliveira MT, Furlaneto-Maia L, Quesada RMB, Furlaneto MC. Hemólise produzida por Candida tropicalis isoladas de amostras clínicas. Rev Soc Bras Med Trop 2010. 43; 318-321. 